\title{
Spatial Diversity Scheme to Efficiently Cancel ISI and ICI in OFDM-OQAM Systems
}

\author{
Nizar Zorba ${ }^{1}$ and Faouzi Bader ${ }^{2}$ \\ ${ }^{1}$ Electrical Engineering Department, Faculty of Engineering, University of Jordan, Amman 11942, Jordan \\ ${ }^{2}$ Centre Technològic de Telecomunicacions de Catalunya, (CTTC), Avenida Carl Friedrich Gauss 7, 08860 Barcelona, Spain
}

Correspondence should be addressed to Nizar Zorba, n.zorba@ju.edu.jo

Received 19 August 2010; Accepted 4 October 2010

Academic Editor: Christos Verikoukis

Copyright (C) 2010 N. Zorba and F. Bader. This is an open access article distributed under the Creative Commons Attribution License, which permits unrestricted use, distribution, and reproduction in any medium, provided the original work is properly cited.

\begin{abstract}
This paper is based on an Offset Quadrature Amplitude Modulation (OQAM) Orthogonal Frequency Division Multiplexing (OFDM) transmission scheme that is operated without a Cyclic Prefix (CP), where the multiple transmitting antennas are employed to substantially reduce the inherent intersymbol and intercarrier interference. The proposed scheme avoids the use of the CDMA technology to get rid of the interference. The nonemployment of the $\mathrm{CP}$ increases the spectral efficiency in comparison with classical CP-OFDM systems, as it does not employ the CP for its correct performance. On the other hand, the non-employment of the CP comes at cost of Intersymbol Interference (ISI). This paper presents a method which cancels the interference terms by employing a multiantenna precoding strategy based on spatial diversity OQAM-OFDM scheme, so that the overall system can get the advantage of the CP removal while no ISI is generated. Moreover, the proposed system benefits from the multiuser gain through an opportunistic scheduler at the transmitter side to select the user with the best channel characteristics at each instant. The resultant scheme OQAM-OFDM-MIMO data rate is obtained in a closed form expression and proved to be higher than the classical CP-OFDM systems.
\end{abstract}

\section{Introduction}

Communication rates in broadband wireless systems achieved very high values in multicarrier techniques, that can be combined with the Multiple-Input-MultipleOutput (MIMO) technology to provide both efficiency and Quality of Service (QoS) to the system. Typically, the channel in these broadband systems is frequency selective, and one of the best multicarrier techniques that can be employed together with MIMO is the Orthogonal Frequency Division Multiplexing (OFDM), because OFDM converts the frequency selective channel into a set of parallel frequency flat channels. Therefore, OFDM is already included in several communication standards as the IEEE 802.11n WLAN standard, while its multiuser OFDM Access (OFDMA) version is already within the IEEE 802.16a/e WiMax standards, the cellular Long-Term Evolution (4GLTE) and the Terrestrial Digital Video Broadcasting (DVB-T) standards $[1,2]$.

OFDM shows some drawbacks that decrease its efficiency. The largest drawback is the requirement for a $\mathrm{CP}$ to tackle the delay in the channel, where the $\mathrm{CP}$ constitutes $10 \%-20 \%$ of the symbol time, with the consequent decrease in the system performance and the invested resources [3]. It also needs a block processing to keep the orthogonality among all the subcarriers, which is a serious problem for scalability, as it is impossible to increase the number of allocated subcarriers because they will be asynchronous with the rest of the block. As all modern communication systems are characterized by users running different applications characterized with various data rates, setup times, and QoS demands, OFDM shows a problem to synchronization in such heterogenous systems. OFDM shows several advantages as its low complexity and its familiarity to both the academia and industry, as it is already implemented in a lot of communication standards. But to further increase the system efficiency, the design of alternative multicarrier schemes is currently under study within the cognitive communications arena $[4,5]$.

An interesting proposal is to modify the OFDM systems by jointly employing it with the Offset Quadrature Amplitude Modulation (OQAM) transmission principle, for what 


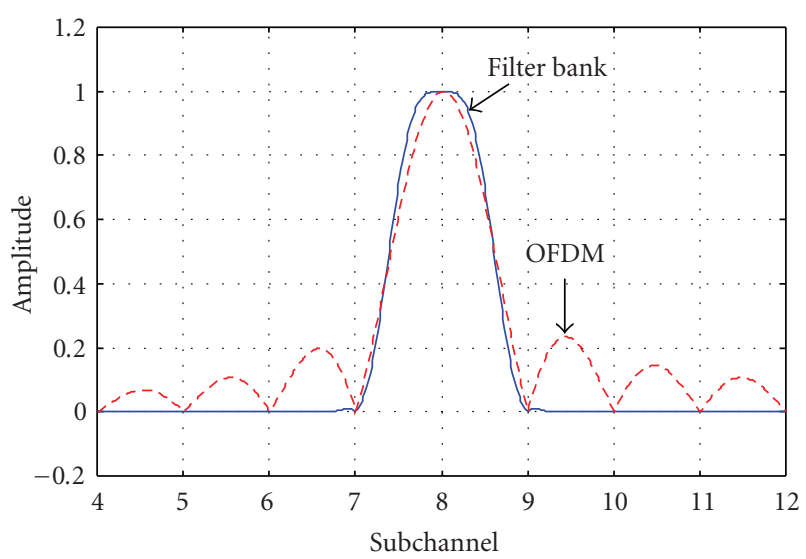

FIGURE 1: The prototype filter advanced basic pulse that reduces the out-of-band frequency leakage.

is known in the literature as the Filter Bank-based Multicarrier (FBMC) transmission [6, 7]. This proposal is shown to enhance the performance and the operational flexibility of OFDM, thanks to its capabilities to exploit the spectral efficiency of filter banks and to enable independence among its subchannels. In OFDM-OQAM, the transmission channel is divided into subchannels, which gives an interesting degree of freedom to control the allocation process, together with the scalability advantage [8]. The main difference of OQAM with respect to OFDM is that instead of rectangular windows, a more advanced basic pulse, also called prototype filter, is used, which can reduce the out-of-band frequency leakage and help to fulfill more stringent spectral masks, as can be seen from Figure 1. The use of OQAM with appropriate filter prototypes allows to obtain an almost negligible ISI and Intercarrier Interference (ICI) assuming that the channel coherence bandwidth is high enough. OFDM-OQAM is mainly characterized by the non-employment of the $\mathrm{CP}$, so that all the system resources are devoted to increase the whole system throughput, thus providing higher spectral efficiency than the classical CP-OFDM [9].

But in OFDM, the CP is employed to avoid the ISI, while OFDM-OQAM removes the CP from its scheme, therefore, the combat of the ISI and the ICI remains as the main challenge in this technique, where these tasks are usually performed by the receiver through some complex operations, representing a main problem for the implementation of this scheme. Therefore, even the study of OFDM-OQAM was initially proposed many years ago [8], but up to now, it has not been included in any commercial standard, mainly due to its large complexity that handicaps its implementation in realistic systems. Recently, an increasing interest in FBMC has again emerged $[4-6,9,10]$, which has to start by finding some signal processing techniques to decrease the OFDMOQAM complexity and to make it commercially viable.

The MIMO technology is already in almost all commercial standards, so that an additional resource is available in the system to be employed to cancel the interference terms without the need for any complex reception techniques and, therefore, to decrease the complexity related to FBMC schemes. Notice that MIMO systems are always proposed to increase the system rate and/or to decrease its error rate, while now, we can face an alternative approach for the implementation of the MIMO systems: the interference cancellation in FBMC. On the other hand, another resource that is employed by the designer to enhance the system behaviour is the multiuser gain, as the availability of multiple users in the system is beneficial to enable the transmitter to select the user with the best channel conditions at each time, and by this way, to increase the system average sum rate. This philosophy is known as the opportunistic scheduler [11], which has already been commercially introduced in the UMTS-HSDPA standard.

Few recent works have been presented to tackle the FBMC systems complexity reduction; the authors in [12] employed the CDMA technology to get rid of the interference without the CP requirement in OFDM-OQAM systems. On the other hand, [13] introduced a MIMO scheme in OFDMOQAM systems when there exist some uncertainty in the Channel State Information (CSI) at the transmitter side, asking for full CSI to operate the system which is a serious handicap in terms of the feedback load.

Up to the authors' knowledge, no previous schemes have been presented in the literature to benefit from the antennas availability to efficiently cancel the ISI and the ICI in the system through a realistic strategy. Therefore, the objective of this paper is to propose a spatial diversity scheme to cancel the ISI and ICI in the system through low-complexity operations, so that the implementation of the OFDMOQAM technique can be possible, with all the presented advantages of the OFDM-OQAM systems. In other words, MIMO will accomplish the required task of interference cancellation in the system that is jointly employed with the system multiuser gain $[11,14]$ to increase the system data rate.

As a summary, the contributions of this work in the field of OFDM-OQAM systems are as follows.

(i) A novel OFDM-OQAM scheme is presented that is operated without the need of the cyclic prefix.

(ii) An intuitive transmission scheme together with lowcomplexity processing at the receiver side are presented within the introduced OFDM-OQAM strategy.

(iii) The multiuser gain in the system is also considered to enhance the system performance, showing better behaviour that the classical CP-OFDM Alamouti [15] technique.

The remainder of this paper is organized as follows: while Section 2 introduces the OFDM-OQAM system applied to single-input single-output systems, Section 3 presents the proposed spatial diversity technique within OFDM-OQAM, where the employed system model and the opportunistic strategy will be explained there. Section 4 will show the numerical results and simulations, followed by Section 5 with the paper conclusions. 


\section{Single-Input Single-Output OFDM-OQAM Approach}

Orthogonal frequency division multiplexing with the cyclic prefix insertion (CP-OFDM) is the most widespread modulation among all the multicarrier modulations, and this is thanks to its simplicity and its robustness against multipath fading using the CP. Nevertheless, this technique causes a loss of spectral efficiency due to the cyclic prefix. Furthermore, CP-OFDM shape is not compact due to the large side lobe levels resulting from the rectangular pulse. This could be avoided by inserting null subcarriers at frequency boundaries in order to avoid harmful interferences into neighboring communication systems. But these practice results in an important loss of spectral efficiency. To avoid these disadvantages, OQAM-OFDM (or FBMC) was proposed as an alternative approach to multicarrier OFDM mainly in cognitive-based networks [16]. In FBMC, there is no need to insert any guard interval. Furthermore, it uses a frequency well-localized pulse shaping such that provides high spectral efficiency [17-20]. Each sub-carrier is modulated with an Offset Quadrature Amplitude Modulation (OQAM) which consists of transmitting the real and the imaginary parts of a complex data symbol with a shift of half the symbol period between them [21].

Because OQAM orthogonality conditions are considered in the real field, the data at the receiver side is carried only by the real (or imaginary) component of the signal. The imaginary (or real) part appears as an intrinsic interference term although the data is always orthogonal to the interference term. But, this term of interference becomes a source of problems when combining OQAM-OFDM with some MIMO techniques.

In both the transmitter and the receiver, a filter bank is obtained by adding to the Fast Fourier Transform (FFT) unit a specific signal processing module: the polyphase network. The number $\mathrm{M}$ of filters in the bank is the size of the FFT and the system is said to have M subchannels, while OFDM has $\mathrm{M}$ subcarriers. The filters are frequency-shifted versions of a prototype low-pass filter, satisfying the Nyquist criterion. A family of prototype filters, which have many desirable properties and can be considered as globally optimal have been designed, using the frequency-sampling technique and a few frequency coefficients [21-23].

The multicarrier filter banks are employed in the transmultiplexer configuration using the Synthesis Filter Bank (SFB) at the transmitter, and the Analysis Filter Bank (AFB) at the receiver [23]. In the FBMC application, the use of critically sampled filter banks would be problematic, since the aliasing effects would make it difficult to compensate imperfections of the channel by processing the subchannel signals after the AFB only. Therefore, a factor of two oversampling is commonly applied in the subchannel signals in the AFB, as shown in Figure 3.

In the current paper, we focused on uniform-modulated filter banks in which the prototype filter $g[\mathrm{~m}]$ of length $L$ is shifted to cover the whole system bandwidth, where the ratio $K=L / M$ is named the overlapping factor because it is the number of multicarrier symbols which overlap in
TABLE 1: Frequency coefficients reference for prototype filters, from [4].

\begin{tabular}{ccccc}
\hline$K$ & $H_{0}$ & $H_{1}$ & $H_{2}$ & $H_{3}$ \\
\hline 2 & 1 & 0.707 & & \\
3 & 1 & 0.911438 & 0.411438 & \\
4 & 1 & 0.971960 & 0.707 & 0.235147 \\
\hline
\end{tabular}

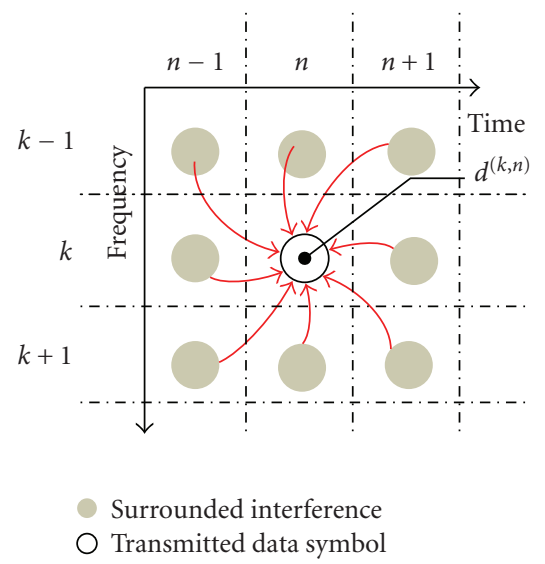

FIGURE 2: Received ISI and ICI from surrounding data symbols.

the time domain. The frequency response of any prototype filters is determined by $K$ samples, as given by Table 1 for several values of the overlapping factor. It must be pointed out that in high speed transmission, the value $K=4$ leads to a good tradeoff between performance and complexity and it is retained as the reference.

Once the prototype filter has been designed, the $M$ filters in the bank are obtained by the frequency shifts $k / M$, with $0 \leq k \leq M-1$. The output signal from the synthesis filter bank is constituted by $M$ subcarriers ( $M=$ IFFT/FFT size) and the set of active subcarriers, $d^{(k, n)}$ denotes the real-valued symbols at the $k$ th subcarrier during the $n$th symbol interval, modulated at rate $2 / T$. The signalling interval $T$ is defined as the inverse of the subcarrier spacing, that is, $T=1 / \Delta f$. The symbols $d^{(k, n)}$ and $d^{(k, n+1)}$ can be interpreted to carry the in phase and quadrature (I/Q) components of the complexvalued symbol $c^{(k, l)}$ (of rate $1 / T$ ) from a QAM-alphabet.

It should be noted that the signs of the $\theta^{(k, n)}\left(\theta^{(k, n)}=\right.$ $j^{k+n}$ ) depicted in the sequence of Figure 3 can be chosen arbitrarily, but the pattern of real and imaginary samples has to follow the shown definition to maintain (near) orthogonality [6]. The synthesized signal burst is, therefore, a composite of multiple subchannel signals each of which consists of a linear combination of time-shifted (by multiples of $T / 2$ ) and overlapping impulse responses of the prototype filter, weighted by the respective symbol values $d^{(k, n)}$. The " $C 2 R$ " and the " $R 2 C$ " blocks in Figure 3 indicate the conversion of the data from complex into real form and the inverse operation, respectively. When a real (imaginary) part of a subcarrier symbol is used, then the unused imaginary (real) part is, at the receiver, a fairly complicated function of surrounding data symbols, as can be seen in Figure 2. We 
TABLE 2: Representation of the time-frequency response of the OFDM-OQAM (FBMC) system considered in this work. Due to the employed offset QAM modulation, the effective time-frequency response will be reduced to only bold values in the table [4].

\begin{tabular}{cccccccccc}
\hline & -4 & -3 & -2 & -1 & 0 & 1 & 2 & 3 \\
\hline-2 & $\mathbf{0}$ & 0.0006 & $-\mathbf{0 . 0 0 0 1}$ & 0 & $\mathbf{0}$ & 0 & $-\mathbf{0 . 0 0 0 1}$ & 0.0006 \\
-1 & 0.0054 & $0.0429 j$ & -0.1250 & $-0.2058 j$ & 0.2393 & $0.2058 j$ & -0.1250 & $-0.0429 j$ & 0.0054 \\
0 & $\mathbf{0}$ & -0.0668 & $\mathbf{0 . 0 0 0 2}$ & 0.5644 & $\mathbf{1}$ & 0.5644 & $\mathbf{0 . 0 0 0 2}$ & -0.668 & 0 \\
1 & 0.0054 & $-0.0429 j$ & -0.1250 & $0.2058 j$ & 0.2393 & $-0.2058 j$ & -0.1250 & $0.0429 j$ & 0.0054 \\
2 & $\mathbf{0}$ & 0.0006 & $-\mathbf{0 . 0 0 0 1}$ & 0 & $\mathbf{0}$ & 0 & $-\mathbf{0 . 0 0 0 1}$ & 0.0006 & $\mathbf{0}$ \\
\hline
\end{tabular}

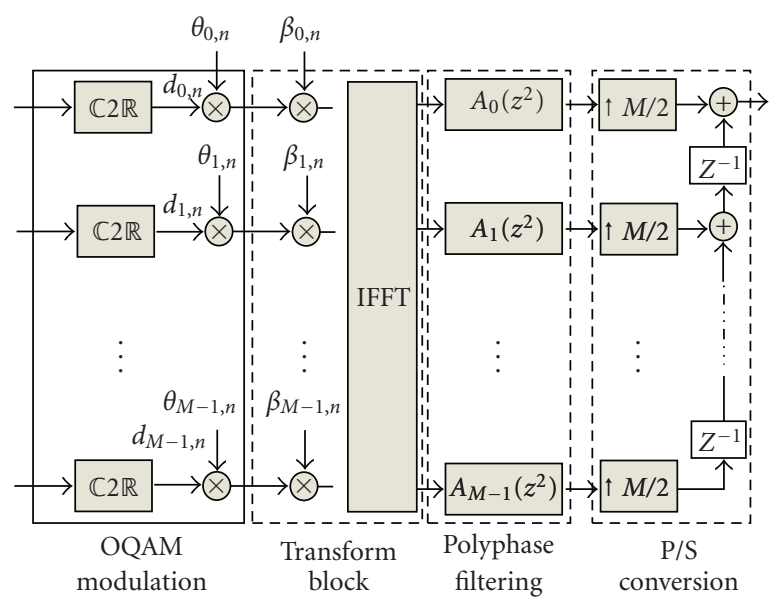

(a)

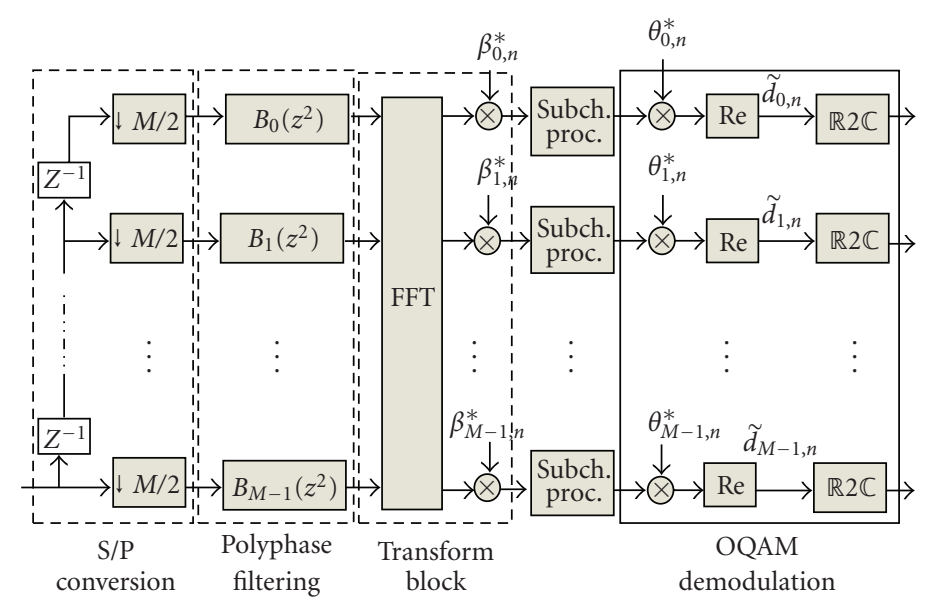

(b)

FIGURE 3: Multicarrier polyphase filter banks for SISO case, (a) synthesis filter banks (SFB), (b) analysis filter banks (AFB).

can write at the transmitter side the baseband equivalent of a discrete time FBMC signal as follows $[21,24]$ :

$$
S[m]=\sum_{k=0}^{M-1} \sum_{n \in z} d^{(k, n)} g^{(k, n)}\left[m-n \frac{M}{2}\right] e^{(2 \pi / M) m(m-D / 2)} e^{j \psi^{(k, n)}}
$$

where $D$ is the delay term and depends on the length of the prototype filter $g[m]$ and $\psi^{(k, n)}$ is an additional phase term. The transmitted symbols $d^{(k, n)}$ are real-valued symbols. Equation (1) can be written in a more compact form as

$$
S[m]=\sum_{M-1}^{k=0} \sum_{n \in z} d^{(k, n)} g^{(k, n)}[m]
$$

where $g^{(k, n)}[m]$ are the shifted versions of $g[m]$ in time and frequency. When the transmitter and the receiver are connected back to back, the signal at the receiver output over the $k$ th sub-carrier and the $n$th time instant is determined using the inner product of $s[m]$ and $g^{(k, n)}[m]$

$$
r^{(k, n)}=\sum_{m=-\infty}^{+\infty} \sum_{k^{\prime}=0}^{M-1} \sum_{n^{\prime} \in z} d^{\left(k^{\prime}, n^{\prime}\right)} g^{\left(k^{\prime}, n^{\prime}\right)}[m] g^{(k, n) \star}[m] .
$$

The coefficients of their impulse responses in the timefrequency domain are illustrated in Table 2. The phase term $\psi^{(k, n)}$ in (1) guarantees and holds the real orthogonality condition

$$
\mathfrak{R e}\left\{\sum_{m=-\infty}^{+\infty} g^{\left(k^{\prime}, n^{\prime}\right)}[m] g^{(k, n) \star}\right\}=\delta^{\left(k, k^{\prime}\right)} \delta^{\left(n, n^{\prime}\right)} .
$$

Considering the SISO FBMC transmission, when passing through the radio channel and adding noise contribution $z^{(k, n)},(3)$ becomes

$$
r^{(k, n)}=h^{(k, n)} d^{(k, n)}+u^{(k, n)}+z^{(k, n)}
$$

where $h^{(k, n)}$ is the channel coefficient at subcarrier $k$ and time index $n, u^{(k, n)}$ is defined as an intrinsic interference and is written as

$$
u^{(k, n)}=\sum_{\left(k^{\prime}, n^{\prime}\right) \neq(k, n)} h^{\left(k^{\prime}, n^{\prime}\right)} d^{\left(k^{\prime}, n^{\prime}\right)} \sum_{m=-\infty}^{+\infty} g^{\left(k^{\prime}, n^{\prime}\right)} g^{(n, k) \star}[m] .
$$

According to Table 2, we note that most part of the energy is localized in a restricted set (shown in bold) around the considered symbol. Consequently, we will assume that the intrinsic interference term depends only on this restricted set (denoted by $k, n$ ) [21]. Moreover, assuming that the channel 
is constant at least over this summation zone, we can write as in $[24]$

$$
\begin{aligned}
& r^{(k, n)} \\
& =h^{(k, n)}(d^{(k, n)}+\underbrace{\sum_{\left(k^{\prime}, n^{\prime}\right) \in \Omega^{(k, n)}} h^{\left(k^{\prime}, n^{\prime}\right)} d^{\left(k^{\prime}, n^{\prime}\right)} \sum_{m=-\infty}^{+\infty} g^{\left(k^{\prime}, n^{\prime}\right)}[m] g^{(k, n) \star}[m]}_{u^{(k, n)}}) \\
& \quad+z^{(k, n)}
\end{aligned}
$$

According to (4), and as $d^{\left(k^{\prime}, n^{\prime}\right)}$ is real valued, the intrinsic interference $u^{(k, n)}$ is pure imaginary. Thus, the demodulated signal can be rewritten as

$$
r^{(k, n)} \approx h^{(k, n)}\left(d^{(k, n)}+j f^{(k, n)}\right)+z^{(k, n)},
$$

where $f^{(k, n)}$ is real-valued. In the case of $n_{t}$ multiple antennas at the transmitter side, we transmit real symbols $d_{i}^{(k, n)}$ at a given time-frequency position $(k, n)$ and at the $i$ th transmit antenna. So, after transmitting through the radio channel, we demodulate at the receiver side as

$$
r^{(k, n)}=\sum_{i=1}^{N_{t}} h_{i}^{(k, n)}\left(d_{i}^{(k, n)}+j f_{i}^{(k, n)}\right)+z^{(k, n)}
$$

where $h_{i}^{(k, n)}$ is the channel coefficient between the $i$ th transmitting antenna and the receiver. A more extended analysis of the multiple antenna case with the proposed ISI and ICI interference suppression scheme is presented in next section.

\section{Spatial Diversity in OFDM-OQAM}

The opportunistic scheduling $[11,14]$ is one of the main transmission techniques in multiuser scenarios, where during the acquisition step, a known training sequence is transmitted from the Base Station (BS). Each one of the users receives the the signal, calculates the received Signalto-Noise Ratio (SNR), and feeds it back to the BS. In order to benefit from the instantaneous channel situation, and, therefore, improving the average system performance, the BS scheduler accomplishes a smart user selection by choosing the user with the largest SNR value for transmission. This opportunistic strategy is proved to be optimal $[11,14]$ as it obtains the maximum rate point.

We consider a multiantenna downlink systems where the transmitter at the BS is provided with $n_{t}$ transmitting antennas, while $V$ receivers exist in the scenario, each one of them equipped with a single-receiving antenna. The case of $n_{t}=2$ is considered along the paper for easiness in the results presentation and to align with all commercial implementations of the IEEE 802.11 pre- $n$ and the proposals for all 4G-LTE systems. Neglecting the $(k, n)$ components to provide a general system model that can be also employed for the Alamouti scheme, the channel $\mathbf{h}_{\left[1 \times n_{t}\right]}=\left[\begin{array}{ll}h_{1} & h_{2}\end{array}\right]$
TABLE 3: OFDM-OQAM proposed setup in a two-antenna scenario.

\begin{tabular}{lcc}
\hline & Subchannel 1 & Subchannel 2 \\
\hline Antenna $_{1}$ & $d_{1}$ on $P P N_{1}$ & $d_{2}$ on $P P N_{2}$ \\
Antenna $_{2}$ & $d_{1}$ on $P P N_{2}$ & $d_{2}$ on $P P N_{1}$ \\
\hline
\end{tabular}

is considered between the transmitting antennas and each one of the users, where a quasistatic block fading model is assumed with independent and identically distributed (i.i.d.) complex Gaussian entries $\sim \mathcal{C} \mathcal{N}(0,1)$. Let $\mathbf{x}=\left[\begin{array}{ll}x_{1} & x_{2}\end{array}\right]^{T}$ be the $n_{t} \times 1$ transmitted vector, while denote $r(v)$ as the received signal at the $v$ th receiver as

$$
\begin{aligned}
r^{(k, n)}(v) & =\mathbf{h}^{(k, n)}(v) \mathbf{x}+z^{(k, n)}(v) \\
& =h_{1}^{(k, n)}(v) x_{1}+h_{2}^{(k, n)}(v) x_{2}+z^{(k, n)}(v),
\end{aligned}
$$

where $z^{(k, n)}(v)$ is an additive Gaussian complex noise component with zero mean and a unit variance. The transmitted signal $\mathbf{x}$ is a coded version of the i.i.d. data symbols $s_{i}$ with $E\left\{\left|s_{i}\right|^{2}\right\}=1$. For ease of notation, the $n$ component is dropped whenever possible to concentrate on the proposed spatial domain processing.

The generated interference in the system due to the nonemployment of the $\mathrm{CP}$ is one of the drawbacks due to considering OFDM-OQAM in current commercial systems. On the other hand, the MIMO technology is already available in almost all OFDM-based wireless standards (e.g., IEEE $802.11 \mathrm{n}$, IEEE $802.16 \mathrm{e} / \mathrm{m}$ and LTE). The MIMO system is mainly devoted to increase the system data rate and/or quality of service, but it can be also employed to accomplish interference mitigation in the system, such mitigation is already known in the research arena through several schemes like space time coding [15] and zero forcing beamforming [25], among others. To apply interference cancellation over OFDM-OQAM systems is a challenging matter due to the time-frequency interference pattern in Table 2, but it would be a very important achievement for the OFDM-OQAM system, as the interference cancellation stands as one of the main milestones to make the OFDM-OQAM to be attractive, avoiding extra interference cancellation complex mechanisms at the receiver side, reducing its complexity and making it suitable for implementation.

The consideration of MIMO with $n_{t}=2$ enables two simultaneous symbols to travel in the channel at the same time and through the same subchannel. Table 3 shows a possible setup for the OFDM-OQAM transmission over 2 subchannels and on a single time instant (i.e., $k=1,2$ and $n$ is dropped from the formulations), where the PPN order is switched over the two antennas.

From Table 3, notice that the two antennas are employed to provide the system with a space-time block coding scheme that will be later employed to cancel the interference at the receiver side through some signal processing. With the shown setup, the second antenna is employed to transmit the same information as in the first antenna, implementing the same principle as the very well-known Alamouti scheme [15]. For sure, some modifications are required to enable 
its application to the OFDM-OQAM technology with its characterizing large amount of generated interference.

Considering this setup, the received signal $r^{(1, n)}$ in the first subchannel states as

$$
r^{(1, n)}=\left(d^{(1, n)}+j f^{(1, n)}\right) h_{1}+\left(d^{(1, n)}+j f^{(1, n)}\right) h_{2}+z^{(1, n)}
$$

where as previously explained, $z^{(1, n)}$ is the noise term received in the subchannel 1 , and $f^{(1, n)}$ accounts for all the interference components [23] that arise from the filterbank usage at the first subchannel, as presented through Table 2. On the other hand, the received signal in the second subchannel is as

$$
r^{(2, n)}=\left(j d^{(2, n)}+f^{(2, n)}\right) h_{1}+\left(j d^{(2, n)}+f^{(2, n)}\right) h_{2}+z^{(2, n)},
$$

with $f^{(2, n)}$ as the interference terms in the second subchannel.

The reader can consider a hypothetical case that $h_{1}$ and $h_{2}$ show equal values in magnitude and opposed in phase, killing the received signal, as the two signals coming from the two antennas will cancel each other, and no signal will reach the receiver. We should remark that this situation has a low probability to happen [15], and even of that, this case would fail in the system outage consideration, exactly as the Alamouti scheme does [15].

3.1. Receiver Processing. Through the proposed transmission scheme, now the receiver has two different arriving signals $r^{(1, n)}$ and $r^{(2, n)}$, one on each subchannel. Note that a whole symbol (i.e., both its real and imaginary parts) is transmitted over two antennas operating on two subchannels (i.e., one single OFDM carrier) and on one time instant, then a full diversity rate [15] is obtained. With the proposed scheme, the two antennas are be efficiently employed in the system to help mitigating the generated OFDM-OQAM interference, thus decreasing the OFDM-OQAM complexity.

At the receiver side, the following low-complexity processing is accomplished to obtain the expression from $r^{(1, n)}$ as

$$
\begin{aligned}
y_{1}= & \operatorname{Re}\left\{h_{1}^{*} r^{(1, n)}+h_{2} r^{(1, n) *}\right\} \\
= & \left(\left|h_{1}\right|^{2}+2 \operatorname{Re}\left(h_{1}^{*} h_{2}\right)+\left|h_{2}\right|^{2}\right) d_{1} \\
& +\operatorname{Re}\left(h_{1}^{*} z^{(1, n)}\right)+\operatorname{Re}\left(h_{2} z^{(1, n) *}\right),
\end{aligned}
$$

where we can see that all the interference terms $f^{(1, n)}$ are removed thanks to the receiver processing. This is a very important step for OFDM-OQAM as the interfering terms have disappeared without the need to complex operations at the receiver side. Unfortunately, some dependence on the channel phase is generated with the receiver processing, due to the $2 \operatorname{Re}\left(h_{1}^{*} h_{2}\right)$ term, that can show positive and negative values depending on the instantaneous channel conditions of both $h_{1}$ and $h_{2}$. A great spatial antenna gain is presented in the system, as the information in $d^{(1, n)}$ is received through both $h_{1}$ and $h_{2}$. Moreover, the data component $d^{(1, n)}$ is received without any other data components, so that with the simple Matched Filter (MF) receiver, the data can be efficiently extracted. Another equation is required for the detection of $d^{(2, n)}$, as the single symbol is composed from its real part $d^{(1, n)}$ and its imaginary part $d^{(2, n)}$, where both parts are compulsory for a correct detection.

The equation for $d^{(2, n)}$ is obtained by applying a different processing for subchannel 2 at the receiver side, to get

$$
\begin{aligned}
y_{2}= & \operatorname{Im}\left\{h_{1}^{*} r^{(2, n)}+h_{2}^{*} r^{(2, n)}\right\} \\
= & \left(\left|h_{1}\right|^{2}+2 \operatorname{Re}\left(h_{1}^{*} h_{2}\right)+\left|h_{2}\right|^{2}\right) d_{2} \\
& +\operatorname{Im}\left(h_{1}^{*} z^{(2, n)}\right)+\operatorname{Im}\left(h_{2}^{*} z^{(2, n)}\right),
\end{aligned}
$$

where we also notice that there is not any interference term in the equation.

The symbol detection, through its $d^{(1, n)}$ and $d^{(2, n)}$ parts, is now solved as no more OFDM/OQAM interfering terms are shown in the previously presented equations. The only remaining problem to be solved is the channel phase effect due to the $\operatorname{Re}\left(h_{1}^{*} h_{2}\right)$ term.

The received SNR for the presented scheme is, therefore, obtained as

$$
\mathrm{SNR}=\frac{1}{2} \frac{\left.|| h_{1}\right|^{2}+2 \operatorname{Re}\left(h_{1}^{*} h_{2}\right)+\left.\left|h_{2}\right|^{2}\right|^{2}}{\left|\operatorname{Re}\left(h_{1}^{*} z^{(1, n)}\right)+\operatorname{Re}\left(h_{2} z^{(1, n) *}\right)\right|^{2}}=\frac{A}{2 B},
$$

where the $1 / 2$ value is due to the separate reception of the two parts of the symbol, therefore, the power of $d^{(1, n)}$ is half the power of the original symbol $s$, which was defined to be unity. Now, we must develop this formulation to see how suitable is the obtained SNR expression and how it can be further improved. Remind that each one of the channel entries (e.g., $h_{1}$ ) is distributed as an i.i.d. complex Gaussian variable $\sim \mathcal{C} \mathcal{N}(0,1)$, so that it has both a real and imaginary components $h_{1}=h_{11}+j h_{12}$ and $h_{2}=h_{21}+j h_{22}$, where each one of these components is distributed as an i.i.d. Gaussian variable $\sim \mathcal{N}(0,1 / 2)$. With these clarifications and under the consideration of i.i.id. noise component, the expression of the denominator can be further developed to obtain

$$
\begin{aligned}
B & =\frac{1}{2}\left(\left|h_{11}\right|^{2}+2 h_{11} h_{21}+\left|h_{21}\right|^{2}+\left|h_{12}\right|^{2}+2 h_{12} h_{22}+\left|h_{22}\right|^{2}\right) \\
& =\frac{1}{2}\left(\left|h_{1}\right|^{2}+2 h_{11} h_{21}+\left|h_{2}\right|^{2}+2 h_{12} h_{22}\right) .
\end{aligned}
$$

Now, considering the numerator $A$ in order to simplify the SNR expression, the value of $2 \operatorname{Re}\left(h_{1}^{*} h_{2}\right)$ can be formulated as

$$
2 \operatorname{Re}\left(h_{1}^{*} h_{2}\right)=2 h_{11} h_{21}+2 h_{12} h_{22}
$$

and, therefore, the SNR expression in (15) stands as

$$
\operatorname{SNR}=\left|h_{1}\right|^{2}+2 \operatorname{Re}\left(h_{1}^{*} h_{2}\right)+\left|h_{2}\right|^{2} \text {. }
$$


Notice that the channel phase effect can be positive or negative, where the value of $2 \operatorname{Re}\left(h_{1}^{*} h_{2}\right)$ has a symmetric distribution over the zero point, as it is only composed of Gaussian $\sim \mathcal{C} \mathcal{N}(0,1)$ variables. Obviously, the receiver is interested in a positive value for the channels phase effect, so that the decoding process is improved.

In order to compare this proposal to current systems, we notice that for a fair comparison, we must consider the Alamouti scheme [15] that is applied over OFDM and following the same power restrictions that are imposed in the system model section. The obtained SNR expression for such strategy [15] is as

$$
\mathrm{SNR}_{\mathrm{alam}}=\left|h_{1}\right|^{2}+\left|h_{2}\right|^{2}
$$

which is shown to provide very good system performance, and it is already included in several broadband wireless communication standards. Comparing the SNR expressions in (18) and (19), we notice that the only difference relates to the channel phase effect $2 \operatorname{Re}\left(h_{1}^{*} h_{2}\right)$ value. As already commented, this value can be either positive or negative with an equally distribution for its sign. If we consider the average performance, then the two SNR expressions will be equal on the long run, but with different Bit Error Rate (BER) behaviour, as the variance of the expression in (18) is larger due to the additional phase term, thus with lower BER performance. This is a severe disadvantage of the proposal up to now, so that some improvement must be accomplished in order to make it more suitable and therefore, not only the $\mathrm{CP}$ gain, the synchronization and the scalability advantages are presents, but also a gain in terms of the rate and BER behaviours. The only problem that remains to solve is the effect of the phase term, as we only require it to show a positive value, where the solution to this problem is obtained through the multiuser gain, as next shown.

When several users exist in the system, the system administrator can benefit from the channel conditions of the available users in the system to select the user with the best channel conditions, for what is known as the multiuser system gain [11]. Several modern wireless communication standards [26] employ such opportunistic scheduling in its operation to increase their performance, and as OFDM/OQAM is targeted to high data rate systems, then we will tackle our objective through the opportunistic scheduler. This objective can be accomplished if we define the opportunistic scheduling as looking to select the user $v$ showing

$$
\max _{v=1: V}\left(\left|h_{1}(v)\right|^{2}+2 \operatorname{Re}\left(h_{1}(v)^{*} h_{2}(v)+\left|h_{2}(v)\right|^{2}\right)\right),
$$

to guarantee that the phase channel effect is always beneficial to the system performance.

On the other hand, the Alamouti scheme can be also operated with an opportunistic scheduler, where the selected user will be the one showing

$$
\max _{v=1: V}\left(\left|h_{1}(v)\right|^{2}+\left|h_{2}(v)\right|^{2}\right),
$$

where the Alamouti scheme is proved [27] to highly benefit from the multiuser gain.

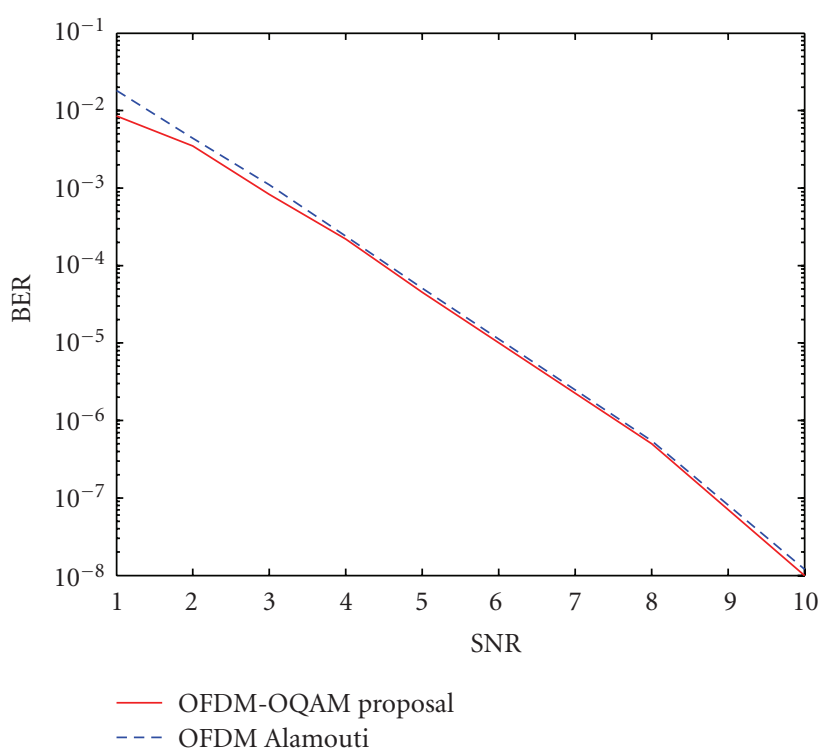

Figure 4: BER performance of Classical OFDM Alamouti and the OFDM-OQAM proposal, both in a multiuser scenario.

It remains to confirm that our proposal outperforms the Alamouti one, and this can be accomplished if we prove that the sign of the phase effect is always positive. We do not concern about the phase distribution, but only about its sign distribution. Notice that the opportunistic selection in the OFDM/OQAM proposal will offer better performance as long as the user with best channel characteristics shows a positive value for the phase effect. The probability $P$ to achieve such situation [28] can be easily formulated as

$$
P=1-\left(\frac{1}{2}\right)^{V-1}
$$

where as expected, increasing the number of available users $V$ will drive an improvement in the probability of better performance of the OFDM/OQAM proposal in comparison to the Alamouti scheme.

\section{Simulations}

To see the behaviour of the proposed scheme, extensive computer simulations are run on a wireless scenario with $n_{t}=2$ transmitting antennas and a variable number of users each one equipped with a single antenna. The transmitter is implementing a spatial diversity scheme over OFDMOQAM, where a total transmitted power $P_{t}=1$ is assumed. A total system bandwidth of $1 \mathrm{MHz}$ is considered in this scenario.

In a first comparison, we consider the BER performance of the proposed scheme in comparison to the standard OFDM Alamouti strategy, both operated in an opportunistic multiuser scenario with 10 users, where the BS carries out the scheduling of the user with the best channel conditions following the selection algorithms in Section 3.1. The results are shown in Figure 4 where both techniques present a very similar behaviour and slightly better for the OFDM-OQAM 


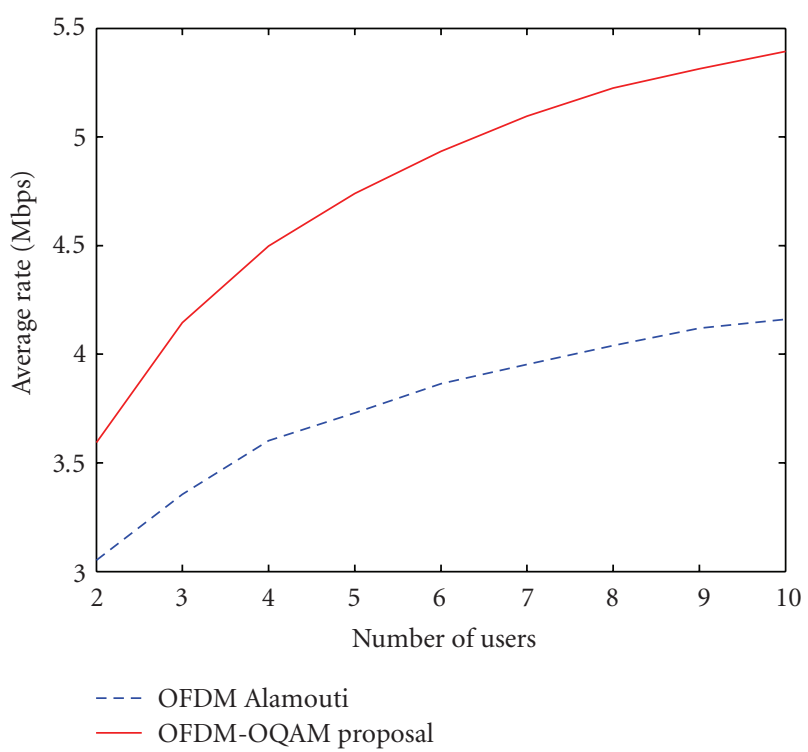

Figure 5: Rate performance of classical OFDM and OFDMOQAM, both operated in the spatial diversity philosophy and within a multiuser scenario.

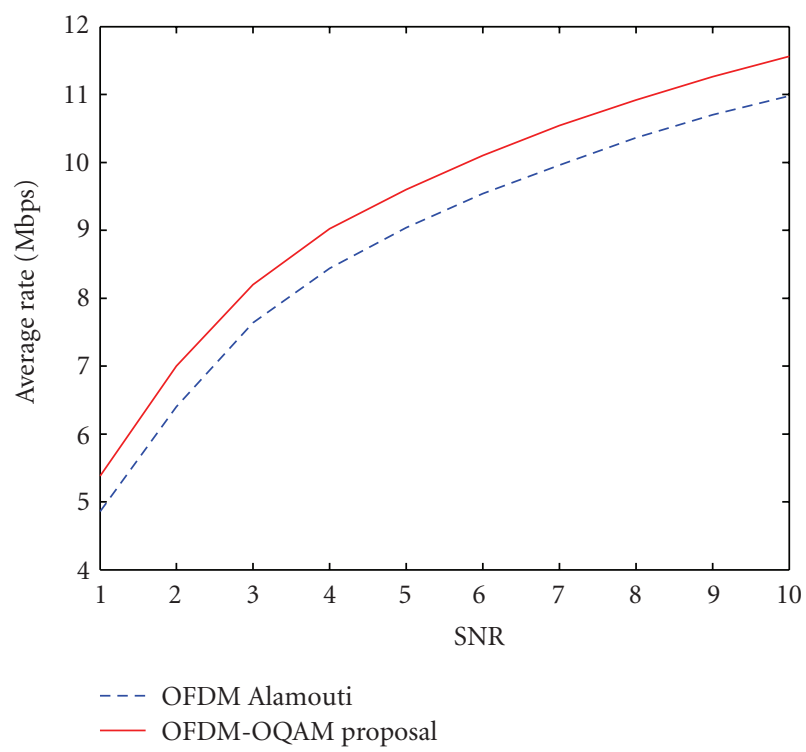

FIGURE 6: Rate performance of classical OFDM and OFDMOQAM, both operated in the spatial diversity philosophy and with a variable SNR value.

proposal. This indicates that the reception quality is very close between the two schemes, which is an encouraging aspect, as all the benefits from the OFDM-OQAM system come at no cost on the reception quality.

One of these improvements is shown in Figure 5, where a scenario with a variable number of users is simulated. The comparison between the classical OFDM Alamouti scheme and the proposed OFDM-OQAM shows the better performance of our proposal, obtaining a higher benefit from the multiuser system capabilities. Obviously, this gain comes

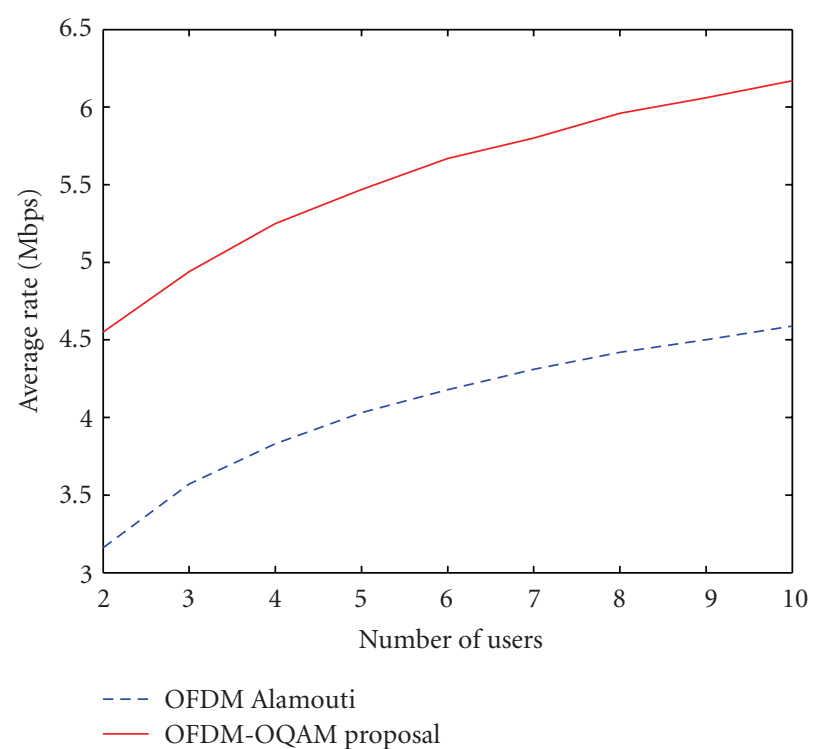

FIGURE 7: Rate performance of classical OFDM and OFDMOQAM, both operated in the spatial diversity philosophy and within an Outdoor multiuser scenario.

from the resource saving as the OFDM-OQAM proposal does not employ the CP, that is assumed to be $12.5 \%$ which is the most typical value within the in commercial standards [3]. But also, the multiuser gain is employed in a better approach by the OFDM-OQAM proposal, as shown in Section 3.1.

To check for the system performance under different values of average SNR, we fix the number of users and vary in the SNR value to check for the proposal behaviour within different scenario conditions. Figure 6 shows a comparison between the proposed scheme and the standard Alamouti strategy applied over the same scenario for a fair comparison. The results show how both schemes have almost the same data rate enhancement for an increase in the SNR operating value, but all the time, being the OFDM-OQAM proposal larger than the standard Alamouti technique.

The last consideration for different scenarios to check for our proposal is in an outdoors channel, as both OFDM and the MIMO technologies have been also proposed for outdoor channels. The channel model for outdoor urban scenarios can be modeled as single or two cluster scenarios, where the channel is regarded as a large dominant component affected by multipath propagation, so that there exists a considerable angle spread at each receiving end. Moreover, the direction of arrival does not necessary match with the exact user position due to the reflections that each signal undergoes. The paper adapts a single-cluster outdoor channel model [29] with an angle spread value of $A S=10$ degrees, and the results are shown in Figure 7. As we can see from the figure, the proposed OFDM-OQAM also outperforms the Alamouti scheme in outdoor scenarios, where the performance of both strategies are improved due to the more directionality that appears in the outdoors channels, and as the BS can select the user with best channel characteristics, then a better 
directionality search is performed. From all the above figures, we realize that our OFDM-OQAM proposal outperforms the Alamouti strategy, and without the need of the CDMA spreading codes proposed in [12].

\section{Conclusions}

A spatial diversity scheme over OFDM-OQAM is presented along the paper to enable the implementation of OFDOQAM in realistic systems. This is thanks to the required low-complexity processing at the receiver side that allows to totally cancel the OFDM-OQAM generated interference. In order to enhance the system data rate and BER performance, a selection of the user with best channel conditions at each transmitting instant is performed through an opportunistic scheduler at the transmitter side. To the authors' knowledge, no previous proposals have been presented in the literature to deal with such scenario setup.

The paper formulated the results of the proposed scheme in terms of data rate and BER and mathematically obtained the SNR expression. The results indicate that OFDM-OQAM proposal stands as a potential alternative to the classical OFDM for its consideration in realistic systems. Moreover, the proposed scheme does not employ the $\mathrm{CP}$, which is a further increase in the system efficiency. Its advantages in terms of scalability and synchronization can be also attractive for the system. Therefore, OFDM-OQAM can be employed in certain scenarios upon the requirements and restrictions for the system designer.

\section{Acknowledgment}

This work was partially supported by the European ICT2008-211887 project PHYDYAS, COST Action IC0902, Generalitat de Catalunya under grant 2009-SGR-940 and Jordan University project (DAR162). This work was partially presented at the International MobiLight conference, Athens, Greece, 2009.

\section{References}

[1] K. Fazel and S. Kaiser, Multi-Carrier and Spread Spectrum Systems, John Wiley \& Sons, New York, NY, USA, 2003.

[2] K. Baum, B. Classon, and P. Sartori, Principles of Broadband OFDM Cellular System Design, Wiley-Blackwell, Hoboken, NJ, USA, 2009.

[3] IEEE 802.16e-2005, "IEEE Standard for Local and Metropolitan Area Networks Part 16," 3GPP, 2006.

[4] "Physical layer for dynamic access and cognitive radio," European Project ICT 211887, PHYDYAS, http://www.ictphydyas.org.

[5] European COST Action IC0902, "Cognitive Radio and Networking for Cooperative Coexistence of Heterogeneous Wireless Networks," January 2010, http://newyork.ing.uniroma1 .it/IC0902.

[6] M. Bellanger, "Transmit diversity in multicarrier transmission using OQAM modulation," in Proceedings of the $3 \mathrm{rd}$ International Symposium on Wireless Pervasive Computing (ISWPC '08), pp. 727-730, Santorini, Greece, May 2008.
[7] M. Bellanger, T. Ihalainen, and M. Renfors, "Filter bank based cognitive radio physical layer," in Proceedings of the ICT Mobile and Wireless Communications Summit (ICT-Mobile Summit'09), Santander, Spain, June 2009.

[8] B. Hirosaki, "A maximum likelihood receiver for an orthogonally multiplexed QAM system," IEEE Journal on Selected Areas in Communications, vol. 2, no. 5, pp. 757-764, 1984.

[9] M. El Tabach, J.-P. Javaudin, and M. Hélard, "Spatial data multiplexing over OFDM/OQAM modulations," in Proceedings of the IEEE International Conference on Communications (ICC '07), pp. 4201-4206, Glasgow, UK, June 2007.

[10] T. Fusco and M. Tanda, "Blind frequency-offset estimation for OFDM/OQAM systems," IEEE Transactions on Signal Processing, vol. 55, no. 5, pp. 1828-1838, 2007.

[11] P. Viswanath, D. N. C. Tse, and R. Laroia, "Opportunistic beamforming using dumb antennas," IEEE Transactions on Information Theory, vol. 48, no. 6, pp. 1277-1294, 2002.

[12] Ch. Lele, P. Siohan, and R. Legouable, "The Alamouti Scheme with CDMA-OFDM/OQAM," EURASIP Journal on Advances in Signal Processing, vol. 2010, Article ID 703513, 2010.

[13] I. Estella, A. Pascual, and M. Payaro, "OFDM and FBMC performance comparison for multistream MIMO systems," in Proceedings of the Future Networks Summit, Florence, Italy, June 2010.

[14] R. Knopp and P. A. Humblet, "Information capacity and power control in single-cell multiuser communications," in Proceedings of the IEEE International Conference on Communications (ICC'95), pp. 331-335, Seattle, Wash, USA, June 1995.

[15] S. M. Alamouti, "A simple transmit diversity technique for wireless communications," IEEE Journal on Selected Areas in Communications, vol. 16, no. 8, pp. 1451-1458, 1998.

[16] B. Farhang-Boroujeny and R. Kempter, "Multicarrier communication techniques for spectrum sensing and communication in cognitive radios," IEEE Communications Magazine, vol. 46, no. 4, pp. 80-85, 2008.

[17] H. Zhang, D. Le Ruyet, D. Roviras, Y. Medjahdi, and H. Sun, "Spectral efficiency comparison of OFDM/FBMC for uplink cognitive radio networks," EURASIP Journal on Advances in Signal Processing, vol. 2010, Article ID 621808, 2010.

[18] M. Shaat and F. Bader, "Low complexity power loading scheme in cognitive radio networks: FBMC capability," in Proceedings of the IEEE International Symposium on Personal, Indoor and Mobile Radio Communications (PIMRC '09), Tokyo, Japan, September 2009.

[19] H. Zhang, D. L. Ruyet, and M. Terre, "Spectral efficiency analysis in OFDM and OFDM/OQAM based cognitive radio networks," in Proceedings of the IEEE Vehicular Technology Conference (VTC '09), Barcelona, Spain, April 2009.

[20] P. Siohan, C. Siclet, and N. Lacaille, "Analysis and design of OFDM/OQAM systems based on filterbank theory," IEEE Transactions on Signal Processing, vol. 50, no. 5, pp. 1170-1183, 2002.

[21] M. Bellanger, Filter Banks, Digital Processing and Signals: Theory and Practice, chapter 11, John Wiley \& Sons, New York, NY, USA, 3rd edition, 2000.

[22] A. Viholainen, T. Ihalainen, T. H. Stitz, M. Renfors, and M. Bellanger, "Prototype filter design for filter bank multicarrier transmission," in Proceedings of the European Signal Processing Conference (EUSIPCO '09), Glasgow, UK, August 2009.

[23] M. G. Bellanger, "Specification and design of a prototype filter for filter bank based multicarrier transmission," in Proceedings of the IEEE International Conference on Acoustics, Speech, and Signal Processing (ICASSP '01), pp. 2417-2420, Salt Lake City, Utah, USA, May 2001. 
[24] C. Lélé, J.-P. Javaudin, R. Legouable, A. Skrzypczak, and P. Siohan, "Channel estimation methods for preamble-based OFDM/OQAM modulations," in Proceedings of the European Wireless Conference (EW'07), Paris, France, April 2007.

[25] T. Yoo and A. Goldsmith, "On the optimality of multiantenna broadcast scheduling using zero-forcing beamforming," IEEE Journal on Selected Areas in Communications, vol. 24, no. 3, pp. 528-541, 2006.

[26] 3GPP, "Practical aspects of multiple architectures for HSDPA," Tech. Rep. TSGR1\#16 (00)1219, 2000.

[27] J. Akhtar and D. Gesbert, "Extending orthogonal block codes with partial feedback," IEEE Transactions on Wireless Communications, vol. 3, no. 6, pp. 1959-1962, 2004.

[28] M. R. Spiegel, Theory and Problems of Probability and Statistics, McGraw-Hill, New York, NY, USA, 1992.

[29] D. Gesbert, L. Pittman, and M. Kountouris, "Transmit correlation-aided scheduling in multiuser MIMO networks," in Proceedings of the IEEE International Conference on Acoustics, Speech and Signal Processing (ICASSP '06), pp. 249-252, Toulouse, France, May 2006. 

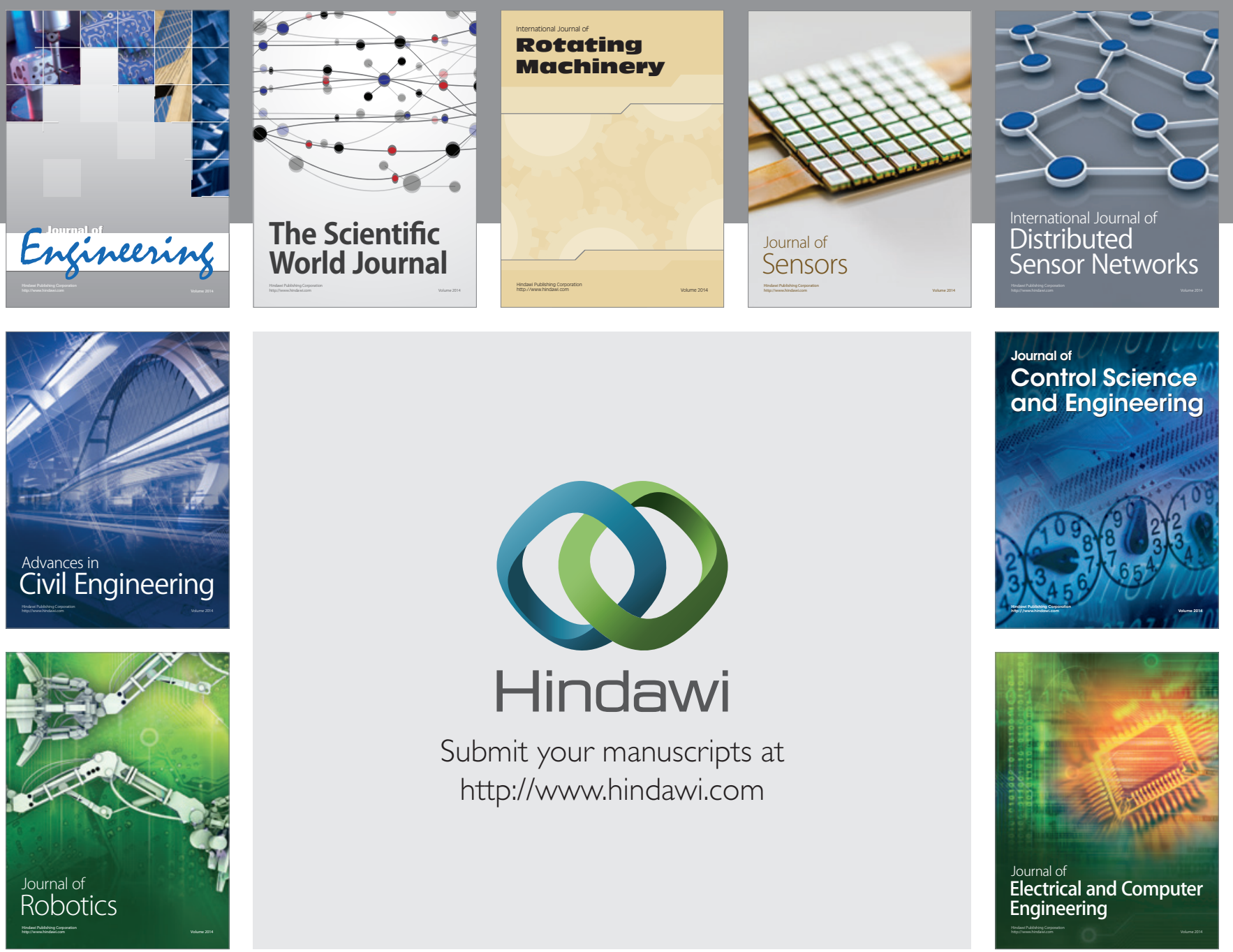

Submit your manuscripts at

http://www.hindawi.com
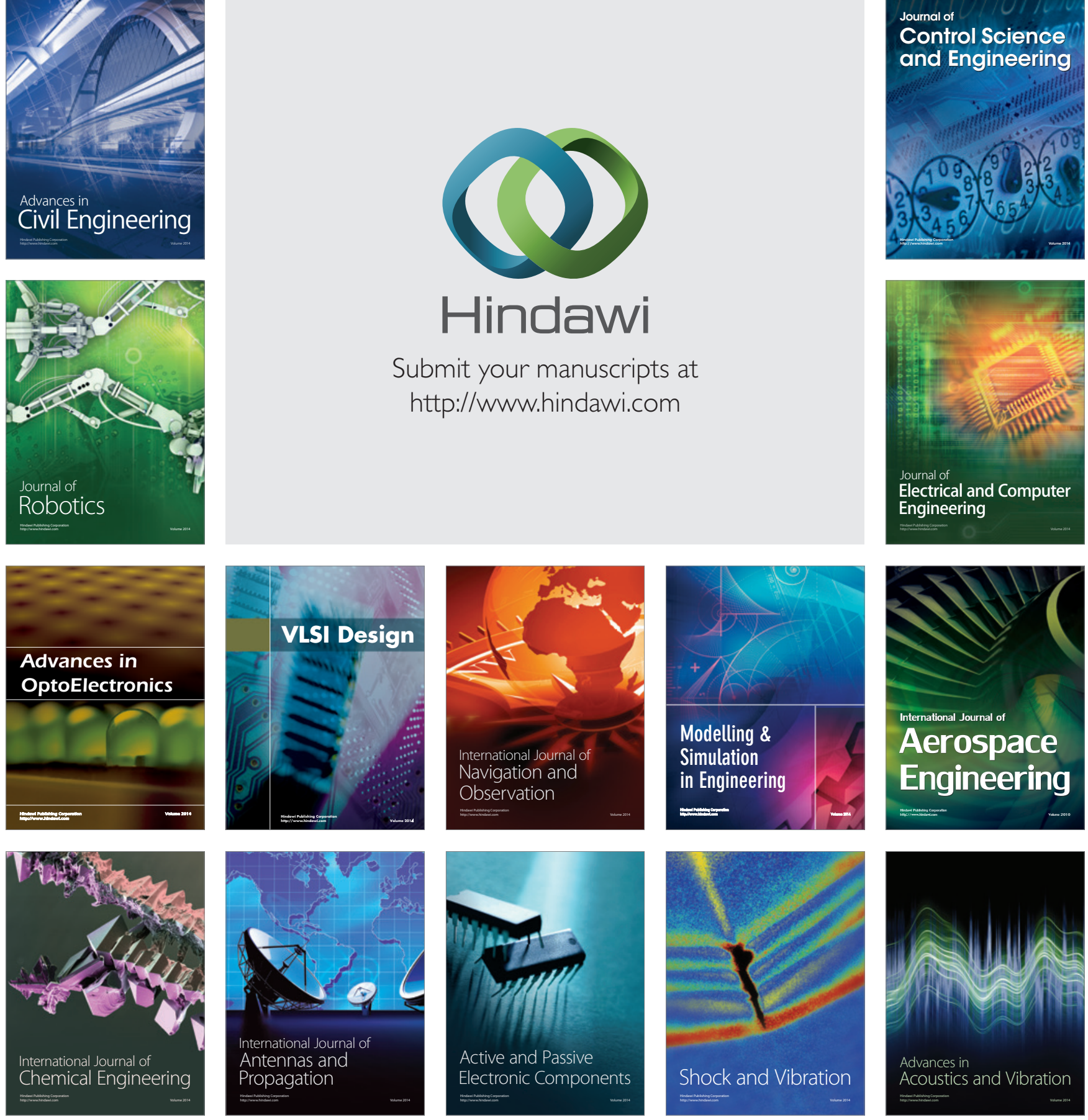